\title{
Magnetopause surface oscillation frequencies at different solar wind conditions
}

\author{
F. Plaschke ${ }^{1}$, K.-H. Glassmeier ${ }^{1,2}$, D. G. Sibeck ${ }^{3}$, H. U. Auster ${ }^{1}$, O. D. Constantinescu ${ }^{1}$, V. Angelopoulos ${ }^{4}$, and \\ W. Magnes ${ }^{5}$ \\ ${ }^{1}$ Institut für Geophysik und extraterrestrische Physik, TU Braunschweig, Braunschweig, Germany \\ ${ }^{2}$ Max-Planck-Institut für Sonnensystemforschung, Katlenburg-Lindau, Germany \\ ${ }^{3}$ NASA, Goddard Space Flight Center, Greenbelt, MD, USA \\ ${ }^{4}$ Institute of Geophysics and Planetary Physics, University of California, Los Angeles, CA, USA \\ ${ }^{5}$ Space Research Institute, Austrian Academy of Sciences, Graz, Austria
}

Received: 3 September 2009 - Revised: 26 November 2009 - Accepted: 30 November 2009 - Published: 11 December 2009

\begin{abstract}
Statistical analyses of the magnetopause (MP) motion observed by THEMIS suggested that the MP oscillates preferably at some prominent (sometimes called "magic") frequencies, which were found to stand out also in ground-based and ionospheric measurements of geomagnetic ultra-low frequency pulsations. In this paper we present an extension to these statistical analyses of the observed MP oscillations examining their dependence on the prevalent interplanetary magnetic field (IMF), solar wind (SW) flow speed and cone angle conditions as well as their local time of occurrence. Our results show enhanced oscillation activity at these frequencies in the noon local time sector during periods of northward IMF, slow or moderate SW speed and low SW cone angle. This combination of conditions supports an interpretation in terms of standing Alfvénic KruskalSchwarzschild surface modes on the MP.
\end{abstract}

Keywords. Magnetospheric physics (Magnetopause, cusp, and boundary layers; Solar wind-magnetosphere interactions)

\section{Introduction}

The magnetopause (MP) is the boundary which separates solar wind (SW) particles and magnetic fields from their magnetospheric counterparts. Its average location, around which boundary oscillations take place, is well characterized by the pressure balance between the total pressure on the magnetosheath side exerted by the impinging SW and the magnetic pressure on the magnetospheric side of the boundary (e.g.,

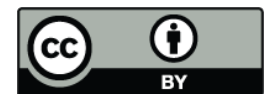

Correspondence to: F. Plaschke (f.plaschke@ @u-bs.de)
Spreiter et al., 1966; Aubry et al., 1970). It was early noted that this location depends on the strength and orientation of the interplanetary magnetic field (IMF). In-situ observations of the MP during different SW conditions made the modelling of its shape and location possible (e.g., Fairfield, 1971; Sibeck et al., 1991; Shue et al., 1997).

The location of the MP is, however, far from being static. Changes in the dynamic pressure of the constantly buffeting SW, the highly turbulent magnetosheath and intrinsic boundary instabilities provide different mechanisms accountable for the perpetual MP motion and undulation. Variations in the SW dynamic pressure or frozen-in IMF can produce MP motion on time scales ranging from seconds to hours (e.g., Song et al., 1988; Sibeck et al., 1989). Kelvin-Helmholtz instability generated surface waves are thought to display periodicities of minutes (Southwood, 1968; Fujita et al., 1996). The same applies to boundary motion produced by reconnection or the reconnection related flux transfer events (FTEs) (Russell and Elphic, 1978; Lockwood and Wild, 1993).

First MP boundary velocity estimations could be obtained from single spacecraft measurements (e.g., Aubry et al., 1971) by combination of the minimum variance (Sonnerup and Cahill, 1967) and deHoffmann-Teller frame analyses or by the minimum Faraday residue technique (Khrabrov and Sonnerup, 1998). Direct determination of the MP velocity, however, is only possible when at least two spacecraft are present in the area of motion. Hence, the ISEE satellite pair provided the first measurements from which this velocity could be reliably estimated using timing techniques (Elphic and Russell, 1979; Berchem and Russell, 1982; Phan and Paschmann, 1996); similar techniques were applied to data from the Interball 1 and Magion 4 satellite pair (Sibeck et al., 2000). Years later measurements from the four CLUSTER spacecraft, which are flying in quasi-tetrahedral

Published by Copernicus Publications on behalf of the European Geosciences Union. 
configuration, allowed for a three-dimensional determination of the boundary velocity vectors (e.g., Haaland et al., 2004; Paschmann et al., 2005), which are snapshots of the MP motion. Unfortunately, a spatio-temporal estimation of this motion (MP tracking) over extended periods of time remained restricted to a few cases where the empirical reconstruction method introduced by De Keyser (2005) or other similar methods could be used.

The new Time History of Events and Macroscale Interactions during Substorms (THEMIS) mission (Angelopoulos, 2008) facilitates for the first time direct MP motion reconstruction from subsequent crossing observations at several radially separated positions in space. During its early coast phase between February and September 2007 the five THEMIS spacecraft were flying in a row ("string of pearls" configuration) on extremely similar near-equatorial and highly elliptical orbits. Since the orbit apogees lay beyond the sub-solar bow shock, the THEMIS spacecraft traversed the dayside MP several thousand times with interspacecraft separations of up to a few thousand kilometers. This particular configuration of five radially aligned spacecraft in the area of MP motion is virtually ideal to reconstruct this motion using the observed MP-spacecraft crossing positions and times as supporting points.

In a case study Glassmeier et al. (2008) used spline functions to interpolate between successive MP crossings in order to infer the MP position at inter-crossing times and compare its motion with magnetosheath density variations. Plaschke et al. (2009a) presented a statistical survey of MP undulation and motion characteristics based on the THEMIS coast phase measurements. They inferred velocity, amplitude and period characteristics from interpolations. A follow-up study (Plaschke et al., 2009b) found that the MP oscillations showed characteristic frequencies, which coincide with a set of stable and recurrent, sometimes called "magic" frequencies, which were first observed in high latitude ionospheric radar measurements and later in ground-based magnetometer measurements (Samson et al., 1992; Francia and Villante, 1997).

The regularity of the sequence of these frequencies suggests them to be fundamental eigenfrequencies of the magnetospheric system. Hence, their appearance has usually been attributed to magnetospheric cavity or waveguide modes. These modes are thought to be confined within a region delimited in radial direction by the static magnetopause and a turning point for compressional mode waves inside the magnetosphere (Harrold and Samson, 1992). It is, however, disillusioning that no final observational evidence has been found so far confirming their existence (Sarafopoulos, 2005). Another possible interpretation has more recently been proposed by Kepko et al. (2002) and Kepko and Spence (2003), who found that some length scales of SW density fluctuations appear more frequently than others. These length scales map to certain characteristic frequencies due to the constant SW flow towards the Earth. Hence, they claim that these SW den- sity fluctuations directly drive geomagnetic pulsations at certain frequencies inside the magnetosphere (e.g., Viall et al., 2008).

Plaschke et al. (2009b) proposed a third possible interpretation: They suggest that Alfvénic MP surface waves generated by mode coupling from magnetosheath compressional disturbances may develop into standing Alfvén waves (or Kruskal-Schwarzschild modes) on the boundary due to reflection on the northern and southern ionospheres. The geometry of the MP boundary would determine in this case a set of eigenfrequencies at which it is able to oscillate preferentially.

The possibility of mode conversion between compressional magnetosheath fluctuations and Alfvén waves running along the MP has been discussed by Belmont et al. (1995) and later by De Keyser et al. (1999). They presented analytical considerations and numerical computations of solutions in the magnetohydrodynamic (MHD) regime for incident waves on the MP under different conditions on both sides and found, that under various conditions resonant amplification can occur. This effect is similar to the mode conversion mechanism discussed by Southwood (1974) describing the resonant mode coupling as part of the field line resonance theory. Within the same theoretical framework the response of the MP to realistic magnetosheath broad band spectra of incident low-frequency waves has been treated by De Keyser and Čadež (2001). Their results confirm and explain the fluctuation amplitude enhancements inside the MP transition region (e.g., Rezeau et al., 1989) as well as the relatively low fluctuation level on the magnetospheric side.

The propagation of boundary surface waves in the framework of ideal MHD theory has first been discussed by Kruskal and Schwarzschild (1954) and was later revisited by Hasegawa and Chen (1974). They examined the situation of a planar boundary between two different plasma regimes, across which magnetic field strength, plasma density and pressure jump, such that the total pressure is conserved. MHD equations are solved after linearization with a plane wave ansatz, which decays exponentially away from the boundary. As boundary conditions the continuity of the normal plasma velocity and of the scalar pressure are used. A stable solution for the surface or Kruskal-Schwarzschild (KS) waves can be found satisfying the dispersion relation:

$\omega=k_{z} \sqrt{\frac{B_{0}^{2}+B_{1}^{2}}{\mu_{0}\left(\rho_{0}+\rho_{1}\right)}}$

where $B$ and $\rho$ denote the magnetic field strength and plasma density in the two plasma half-spaces, $\omega$ is the angular frequency and $k_{z}$ the wave vector component along the magnetic field. The tension of the magnetic flux tubes acts as restoring force. Field aligned currents associated with its propagation may close in the ionospheres, where the waves may be reflected to set up a standing surface wave on the MP. 
The aim of this paper is to address the question if the observed MP oscillation frequency distribution displaying the prominent frequencies may have been caused by SW pressure variations, reconnection related phenomena such as FTEs or if it is a feature caused by the MP itself, either via MP eigenoscillations or intrinsic instabilities (e.g. KelvinHelmholtz).

\section{Data and methods}

The set of MP oscillation half periods presented in Fig. 2 of Plaschke et al. (2009b) is the starting point of our investigation: Fluxgate Magnetometer (FGM, see Auster et al., 2008) and Electrostatic Analyzer (ESA, see McFadden et al., 2008) spin frequency sampled data, measured onboard the five THEMIS spacecraft during their early coast phase (between February and September 2007), were manually inspected to identify sequences of MP-spacecraft crossings. In particular we looked for rotations in the magnetic field temporally coinciding with characteristic changes of its modulus and of the particle (electron and ion) energy flux spectra between magnetospheric and magnetosheath levels. Thereby, 6697 MP crossings were selected in total, where both FGM and ESA data had to be available for their identification. Figure 1 exemplarily shows a sequence of MP crossings detected on four of the five THEMIS spacecraft between 18:15 and 18:30 UT on 16 June 2007. On that day ThB was the leading and ThA the trailing spacecraft in the coast phase "string of pearls" configuration. As can be seen in the upper five panels of Fig. 1, ThB was the first to cross the MP exiting the magnetosphere, the probes $\mathrm{ThD}$, ThC and ThE followed. After a brief excursion into the magnetosheath these four spacecraft returned to the magnetosphere before the end of the selected interval at 18:30 UT. Only the innermost spacecraft ThA remained in the magnetosphere during the whole interval.

Crossings of the MP boundary are marked with vertical black lines in the magnetograms of Fig. 1. These times and the corresponding positions of the spacecraft have to be further processed before a spline interpolation can be applied to infer the MP position also at inter-crossing times, when it was not observed: The positions of the spacecraft were transformed into an aberrated geocentric solar magnetospheric coordinate system (AGSM). In this coordinate system the $x$ direction is inclined by $5^{\circ}$ from the Earth-Sun line towards the direction of motion of the Earth to align it with the direction of incidence of the SW; $z$ points in the direction of the Earth's magnetic dipole axis and $y$ completes the right handed orthogonal coordinate system.

Spacecraft positions in the AGSM system can now be easily reduced to a one-dimensional equivalent standoff distance

$r_{0}=r\left(\frac{2}{1+\cos \theta}\right)^{-\alpha}$

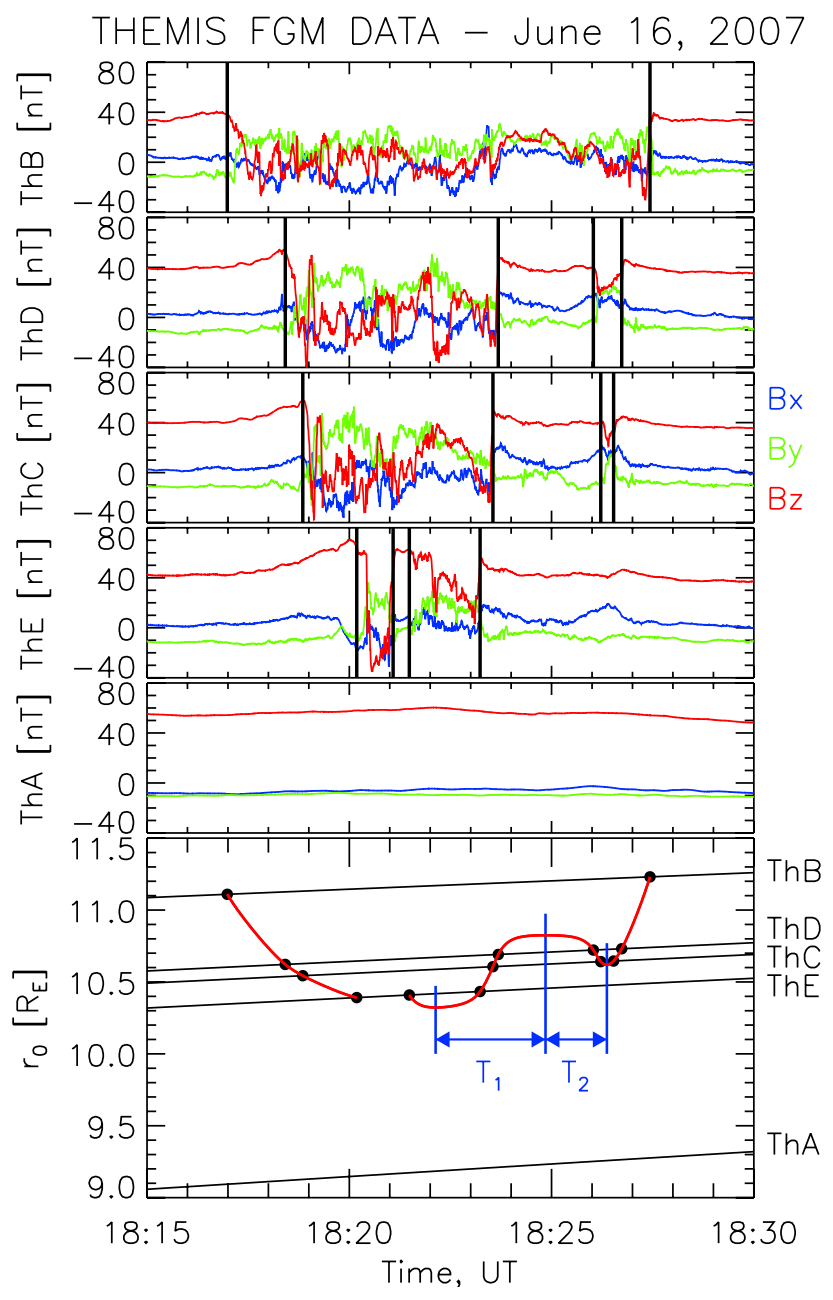

Fig. 1. Top five panels: FGM magnetic field measurements (GSM coordinates) of the five THEMIS spacecraft (ThB, ThD, ThC, ThE and ThA) between 18:15 and 18:30 UT on 16 June 2007. Vertical lines depict crossings out of or into the magnetosphere. Bottom panel: diagonal lines show the equivalent standoff distance $r_{0}$ of the spacecraft. Dots depict the observed crossings, the red lines show the spline interpolations between crossing times and positions.

using the MP shape function of the model introduced by Shue et al. (1997). Here $r$ and $\theta$ denote the radial distance to the probe location from Earth and the angle between this radial line and the AGSM $x$-axis (aberrated Earth-Sun line). The parameter $\alpha$ is set it to a constant value of $\alpha=0.5959$. The result is a set of 6697 equivalent standoff distances $\left(r_{0}\right)$ and the corresponding times of MP observations. This set is divided into subsets of consecutive MP crossings, where the maximum time between two of them is set to $10 \mathrm{~min}$ in order to avoid bridging of too long intervals without supporting points of known MP position with spline interpolation. Furthermore, the subset sequences are checked for soundness: The inward or outward motion of the MP detected at two 
consecutive times should correspond to the relative positions of the detecting spacecraft; else the subset is divided such that this criterion is fulfilled in the two resulting subsets. Two consecutive crossings detected by the same spacecraft may only account for motion reversals; if more than two crossings are observed in succession by the same spacecraft the subset is also further divided to avoid a senseless interpolation of the spacecraft position. If the resulting subsets contain more than $3 \mathrm{MP}$ crossing positions $\left(r_{0}\right)$ and times a spline interpolation is performed using the function pair CURV1 and CURV2 with $\sigma=3$ as described in Cline (1974).

The result of the division into subsets is shown in the bottom panel of Fig. 1: in this panel the equivalent standoff distance of the spacecraft (in units of the Earth radius $R_{E}$ ) is depicted by the diagonal lines. As can be seen, they are moving outward during the interval. The observed MP crossings are marked with black circles. Between 18:20 and 18:24 UT ThE traverses the MP four times. The initial set of crossings is divided into two subsets, from which the first one comprises the first $4 \mathrm{MP}$ crossings (spacecraft sequence: B, D, C, E) and the second one the last 9 crossings (spacecraft sequence: E, E, C, D, D, C, C, D, B). Spline interpolation is performed on both subsets, the result is shown by the red lines. The time intervals between MP motion reversals (extremal points of the second spline interpolation) can now be interpreted as MP oscillation half-periods; they are denoted with $T_{1}$ and $T_{2}$ in Fig. 1. From these full oscillation periods $(2 T)$ and MP oscillation frequencies $(1 /(2 T))$ can be computed. In total 682 MP oscillation half-periods could be obtained. The dataset of these half-periods also comprises the AGSM longitude of observation information $\lambda$, which is computed by averaging of the spacecraft positions (in the AGSM coordinate system) belonging to the corresponding sets of MP crossings.

OMNI high resolution (HRO, sampling period: 1 min) SW data were added to this dataset. They constitute a combination of SW measurements from several spacecraft time-shifted to represent the SW conditions at the bow shock nose. The composition of the OMNI dataset is extensively explained in http://omniweb.gsfc.nasa.gov/html/ HROdocum.html. To take into account the variable SW particle propagation time from the bow shock to the MP under different conditions we opted for extending (and not timeshifting) all half-period intervals by $5 \mathrm{~min}$ precursory intervals to compute averages of the interplanetary magnetic field (IMF) $B_{z}$ (in the GSM coordinate system), SW flow speed $v_{\text {sw }}$ and cone angle $\vartheta_{\text {sw }}$ (defined as the angle between the Sun-Earth line and the IMF direction): Linear interpolations of the respective SW measurements were computed for every second of the selected intervals; afterwards those interpolated values were averaged in a second step. This could, of course, only be performed if the necessary SW data were available, which was the case for 508 half-period determinations. The dataset comprising these MP oscillation events will from now on be denoted as the sample population.
Frequency distributions under different SW conditions and observation longitudes may be obtained from this dataset. Given a probability density function (PDF) for the frequency $P\left(f \mid B_{z}, v_{\mathrm{sw}}, \lambda, \vartheta_{\mathrm{sw}}\right)$ dependent only on $B_{z}, v_{\mathrm{sw}}, \lambda$ and $\vartheta_{\mathrm{sw}}$ the integrated (independent) probability density $P(f)$ is obtained by Bayes theorem:

$$
\begin{aligned}
P(f) & =\iiint P\left(f \mid B_{z}, v_{\mathrm{sw}}, \lambda, \vartheta_{\mathrm{sw}}\right) . \\
& \cdot P\left(B_{z} \cap v_{\mathrm{sw}} \cap \lambda \cap \vartheta_{\mathrm{sw}}\right) \mathrm{d} B_{z} \mathrm{~d} v_{\mathrm{sw}} \mathrm{d} \lambda \mathrm{d} \vartheta_{\mathrm{sw}}
\end{aligned}
$$

where $P\left(B_{z} \cap v_{\mathrm{sw}} \cap \lambda \cap \vartheta_{\mathrm{sw}}\right)$ is the joint probability of the four quantities. Computing frequency histograms from sample population events (or half-period determinations) without any weighting would imply taking not only $P\left(f \mid B_{z}, v_{\mathrm{sw}}, \lambda, \vartheta_{\mathrm{sw}}\right)$ but also $P\left(B_{z} \cap v_{\mathrm{sw}} \cap \lambda \cap \vartheta_{\mathrm{sw}}\right)$ from this small population. The joint PDF, however, can be obtained from the OMNI SW dataset with much higher accuracy: Consecutive 550s averages of $B_{z}, v_{\mathrm{sw}}$ and $\vartheta_{\mathrm{sw}}$, which correspond to the aforementioned $300 \mathrm{~s}$ precursory intervals and $T=250$ s half-period intervals (of typical oscillations with a low frequency of $2 \mathrm{mHz}=1 /(2 T)$ ), were taken for the times between March and September 2007. These averages constitute basic populations for the respective quantities. The reference PDF for the AGSM longitude $\lambda$ should be uniform, since the THEMIS coverage of the dayside and dusk flank regions is equilibrated for the coast phase times (steady orbit rotation).

Figure 2 shows these basic population PDFs for $B_{z}, v_{\mathrm{sw}}$, $\lambda$ and $\vartheta_{\text {sw }}$ (yellow bars) as well as the corresponding sample population distributions (solid lines). Both distributions may be assimilated by proper weighting of the sample population events. Since its sample size is small, only a first order correction may be applied: Thresholds for each of the 4 quantities $\left(\tilde{B}_{z}=0 \mathrm{nT}, \tilde{v}_{\mathrm{sw}}=500 \mathrm{~km} / \mathrm{s}, \tilde{\lambda}=40^{\circ}\right.$ and $\left.\tilde{\theta}_{\mathrm{sw}}=30^{\circ}\right)$ are chosen, which divide the populations into 16 groups. The choice for the $B_{z}$ and $v_{\mathrm{sw}}$ thresholds are motivated from the corresponding basic populations depicted in Fig. 2: $\tilde{B}_{z}=0 \mathrm{nT}$ divides the populations symmetrically (northward and southward IMF events); $\tilde{v}_{\mathrm{sw}}=500 \mathrm{~km} / \mathrm{s}$ divides the populations into low/moderate SW flow velocity events (increased PDF in Fig. 2 panel (b) below $\tilde{v}_{\text {sw }}$ ) and high SW velocity events, which are observed with lower probability of occurrence. In the case of the parameters $\lambda$ and $\theta_{\mathrm{sw}}$ the respective thresholds were selected ensuring an almost symmetric distribution of events on either side of them. Events of the sample population in each of the 16 groups are now weighted by:

$$
\frac{P_{\mathrm{b}}\left(B_{z} \gtrless \tilde{B}_{z}, v_{\mathrm{sw}} \gtrless \tilde{v}_{\mathrm{sw}}, \vartheta_{\mathrm{sw}} \gtrless \tilde{\vartheta}_{\mathrm{sw}}\right) \cdot P_{\mathrm{b}}(|\lambda| \gtrless \tilde{\lambda})}{N\left(B_{z} \gtrless \tilde{B}_{z}, v_{\mathrm{sw}} \gtrless \tilde{v}_{\mathrm{sw}},|\lambda| \gtrless \tilde{\lambda}, \vartheta_{\mathrm{sw}} \gtrless \tilde{\vartheta}_{\mathrm{sw}}\right) / N_{\text {total }}}
$$

where $P_{\mathrm{b}}$ is the occurrence probability for events under certain conditions obtained from the basic population dataset, $N$ (conditions) is the number of events of the sample population in the group (for which the specific conditions hold) and 
A) $B_{\text {ZIMF }}$ occurrence probability density

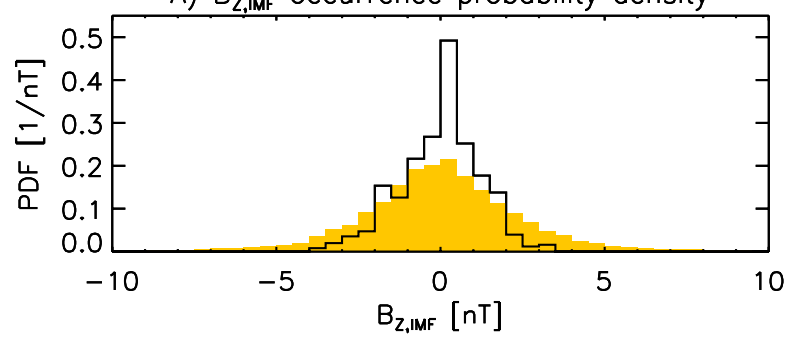

B) $v_{S W}$ occurrence probability density

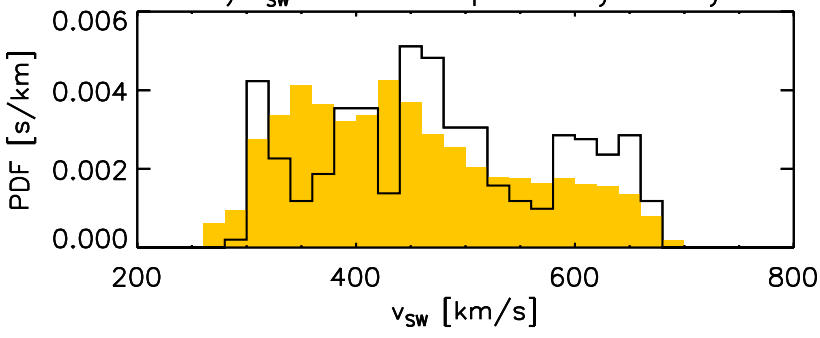

C) $\lambda_{\text {AGSM }}$ occurrence probability density

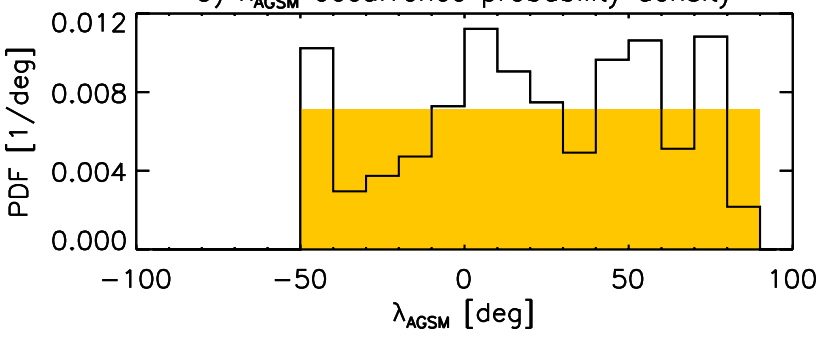

D) $\theta_{\mathrm{SW}}$ occurrence probability density

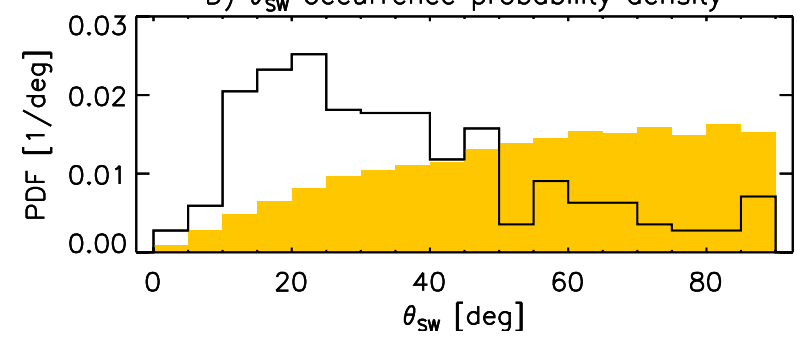

Fig. 2. Probability density functions (PDFs) of events from the halfperiod sample population (solid lines) and the basic population obtained from 7 months of OMNI SW data (yellow bars in panels A, B and D). Distributions are plotted against: (A) IMF $B_{z}$, (B) SW flow speed $v_{\mathrm{sw}}$ (C) AGSM longitude angle $\lambda$ and (D) IMF cone angle $\vartheta_{\text {sw. }}$. In panel (C) the basic population (yellow bars) is given by the constant $\mathrm{PDF}$ in the interval $\left[-50^{\circ}, 90^{\circ}\right]$. It should be noted that the longitude distributions are asymmetric, because the dawn flank magnetospheric regions were not covered by the THEMIS coast phase orbits before the injections into the main phase orbits took place in September 2007.

$N_{\text {total }}=508$ is the sample population size. Corrected conditioned frequency distribution histograms may now be computed by summing up the weighting factors of the conditioncorresponding sample population events.

It should be noted that this correction would be rather small if only the probability discrepancies in the three first quantities would be considered. As can be seen in panels (a), (b) and (c) of Fig. 2 basic population and sample population distributions roughly match (without regard of the fluctuations due to the relatively small number of half-period events available). In panel (d), however, where sample and basic distributions are shown against the IMF cone angle, a systematic discrepancy is visible: The usual cone angle distribution maximizes between $60^{\circ}$ and $90^{\circ}$. The cone angle distribution obtained from the 508 half-period events, instead, maximizes at quite low angles of about $20^{\circ}$. The question why this discrepancy is apparent as well as its implications on the results obtained shall be discussed later on.

In order to approximate the distributions, weights of low/high cone angle events must be significantly reduced/increased. Table 1 shows the occurrence probability for events in both basic $\left(P_{\mathrm{b}}\right)$ and sample $\left(P_{\mathrm{s}}\right)$ populations under the conditions specified; the eighth column of the table indicates the weighting factors applied to the sample population events. The mismatch between the cone angle distributions is reflected in the low weighting factors $\left(P_{\mathrm{b}} / P_{\mathrm{s}}\right)$ for events of low cone angle (rows $2,4,6, \ldots$ ) and the high weighting factors for the events of opposite condition (rows $1,3,5, \ldots$ ). The lowest weighting factors (below 0.3 ) are found for conditions specified in rows 2, 6 and 8; all three groups correspond to northward IMF conditions. The highest weighting factor is applied to group (row) 5 , which corresponds to MP oscillations measured at the flanks during low SW speed and high SW cone angle intervals; these events are underrepresented in the sample population. The last column of the table indicates the number of events in each group.

\section{Results and discussion}

The original frequency distribution as presented in Plaschke et al. (2009b) before further selection is depicted in Fig. 3 (thick solid line). Reduction of this original dataset by extraction of those events, for which no SW data from the OMNI database is available, yields the MP oscillation frequency distribution depicted by the hatched area. This area is completely enclosed by the thick solid line (original distribution) and also resembles the shape of this distribution, because the reduction due to lacking SW data can be regarded as a random extraction of events: main features of the distribution are being conserved. Application of the weighting factors $P_{\mathrm{b}} / P_{\mathrm{s}}$ from Table 1 to the corresponding half-period events yields the distribution depicted by the yellow bars in Fig. 3. For this case the second (right) axis indicates the probability density function (PDF) normalized in the interval ranging from 0 to $6 \mathrm{mHz}$.

Comparing the unweighted and weighted event distributions (hatched and yellow areas in Fig. 3) it can be seen that the latter exhibits much stronger fluctuations. This is related to the fact that some few events, particularly those observed during high IMF cone angle conditions, have to be weighted 
Table 1. Probability of occurrence of events in the basic population $\left(P_{\mathrm{b}}\right.$, column 6$)$ and in the sample population $\left(P_{\mathrm{s}}\right.$, column 7) with the conditions specified in the columns 2 to 5 . The threshold levels are: $\tilde{B}_{z}=0 \mathrm{nT}, \tilde{v}_{\mathrm{sw}}=500 \mathrm{~km} / \mathrm{s}, \tilde{\lambda}=40^{\circ}$ and $\tilde{\vartheta}_{\mathrm{sw}}=30^{\circ}$. The last two columns indicate the weighting factor $P_{\mathrm{b}} / P_{\mathrm{S}}$ applied to the half-period events and the number of events $N$ in the corresponding groups.

\begin{tabular}{|c|c|c|c|c|c|c|c|c|}
\hline \# & $B_{z} \gtrless \tilde{B}_{z}$ & $v_{\text {sw }} \gtrless \tilde{v}_{\text {sw }}$ & $|\lambda| \gtrless \tilde{\lambda}$ & $\vartheta_{\mathrm{sw}} \gtrless \tilde{\vartheta}_{\mathrm{sw}}$ & $P_{\mathrm{b}}$ & $P_{\mathrm{S}}$ & $P_{\mathrm{b}} / P_{\mathrm{s}}$ & $N$ \\
\hline 1 & $>$ & $>$ & $>$ & $>$ & 0.052 & 0.089 & 0.592 & 45 \\
\hline 2 & $>$ & $>$ & $>$ & $<$ & 0.010 & 0.057 & 0.175 & 29 \\
\hline 3 & $>$ & $>$ & $<$ & $>$ & 0.070 & 0.051 & 1.367 & 26 \\
\hline 4 & $>$ & $>$ & $<$ & $<$ & 0.013 & 0.008 & 1.692 & 4 \\
\hline 5 & $>$ & $<$ & $>$ & $>$ & 0.129 & 0.043 & 2.976 & 22 \\
\hline 6 & $>$ & $<$ & $>$ & $<$ & 0.023 & 0.093 & 0.249 & 47 \\
\hline 7 & $>$ & $<$ & $<$ & $>$ & 0.172 & 0.079 & 2.183 & 40 \\
\hline 8 & $>$ & $<$ & $<$ & $<$ & 0.031 & 0.144 & 0.214 & 73 \\
\hline 9 & $<$ & $>$ & $>$ & $>$ & 0.051 & 0.061 & 0.834 & 31 \\
\hline 10 & $<$ & $>$ & $>$ & $<$ & 0.012 & 0.024 & 0.489 & 12 \\
\hline 11 & $<$ & $>$ & $<$ & $>$ & 0.068 & 0.047 & 1.437 & 24 \\
\hline 12 & $<$ & $>$ & $<$ & $<$ & 0.015 & 0.039 & 0.391 & 20 \\
\hline 13 & $<$ & $<$ & $>$ & $>$ & 0.126 & 0.063 & 2.007 & 32 \\
\hline 14 & $<$ & $<$ & $>$ & $<$ & 0.025 & 0.057 & 0.443 & 29 \\
\hline 15 & $<$ & $<$ & $<$ & $>$ & 0.169 & 0.089 & 1.903 & 45 \\
\hline 16 & $<$ & $<$ & $<$ & $<$ & 0.034 & 0.057 & 0.591 & 29 \\
\hline
\end{tabular}

with large coefficients, which in some cases exceed factors of 2 (groups/rows 5, 7 and 13 in Table 1). This results in overestimated occurrence probabilities for the frequency bins to which these few events correspond. Weighting is, however, necessary in order to reduce the effect of the unbalanced distribution of events within parameter space in comparison to the basic population (see Fig. 2).

Although the weighted and unweighted event distributions seem to be quite different, it can be shown that these differences are not significant. On the basis of the unweighted event distribution, as probability density function for the frequency interval from 1 to $6 \mathrm{mHz}$, and of the total number of events of the weighted event distribution in this frequency interval, confidence intervals can be computed for each bin (binomial distribution) to see if both distributions differ significantly. At the $95 \%$ confidence level only one value of the weighted event distribution is outside of its corresponding confidence interval; at the $99 \%$ confidence level all bin values are within the computed confidence intervals. Hence, the distributions depicted in Fig. 3 do not significantly differ from each other, which gives us confidence in our weighting, conducted in order to avoid heavy over or underweighting of some event groups.

Unfortunately, it is not possible to diminish the apparent bias in the lowest frequency part of the distributions depicted in Fig. 3 by weighting. As can be seen, below $1 \mathrm{mHz}$ the relative probability of occurrence is considerably decreased. There are basically two reasons therefor: First, longer MP oscillation periods produce larger gap times between MP crossing observations. However, the largest gap time has been set to $10 \mathrm{~min}$ in order to avoid the bridging of too long time intervals by spline interpolation without further supporting points of known MP position. Consequently, MP oscillations with lowest frequencies are less probably identified; the spectra of Fig. 3 are attenuated below $1 \mathrm{mHz}$. Secondly, the finite residence time in the region of MP motion also reduces the maximal length of identifiable MP oscillation periods. Since the spectrum of oscillations cannot be derived from other than MP motion observations, it is not possible to compute weighting factors in order to correct the bias apparent in the low frequency part. In any case, since the number of observed events there is relatively low, the weighting factors would probably be quite large producing distorted results due to heavy overweighting of a few oscillation events.

As discussed in Plaschke et al. (2009b), the maxima of the original MP oscillation frequency PDF (at 1.3, 1.9, 2.7 and $3.1 \mathrm{mHz}$ with $0.2 \mathrm{mHz}$ bin size) are on or quite close to the fundamental frequencies reported by Samson et al. (1992) (only difference: 3.3 instead of $3.1 \mathrm{mHz}$ ). In order to investigate which source might be primarily responsible for MP oscillations at these frequencies it is sensible to select MP oscillation half-periods with regard to the conditions that were prevalent during the respective times of observation to see if the resulting frequency distributions show an increased occurrence probability at the respective frequency bins. If this is the case, sources for which these conditions are favorable are more likely causing the prominent maxima.

Consequently, we computed frequency PDFs in the range between 0 and $6 \mathrm{mHz}$ for different event selections applying all possible combinations of criteria $\left(B_{z} \gtrless \tilde{B}_{z}, v_{\text {sw }} \gtrless \tilde{v}_{\text {sw }}\right.$, $|\lambda| \gtrless \tilde{\lambda}, \vartheta_{\text {sw }} \gtrless \tilde{\vartheta}_{\text {sw }}$ ) including the non-application of any selection with regard to some or any of the parameters under consideration. From these PDFs the events were counted which belong to the frequency bins $1.3,1.9,2.7$ and $3.3 \mathrm{mHz}$ 


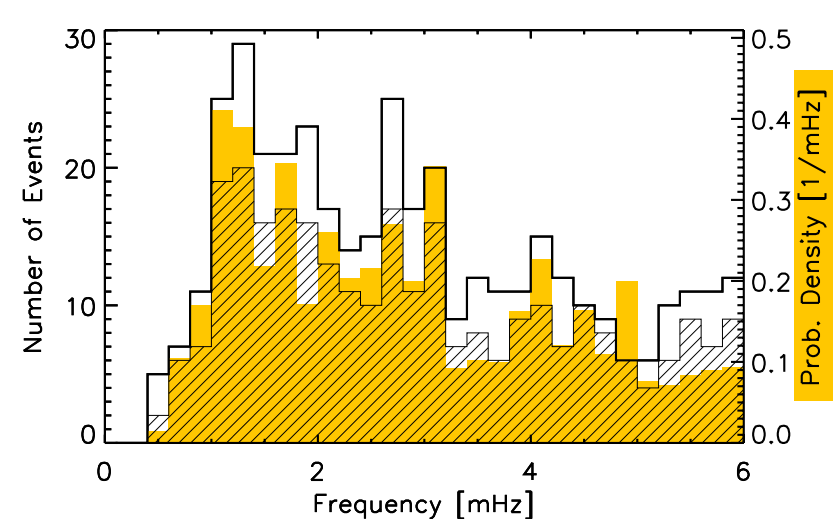

Fig. 3. Number of half-period MP oscillations observed under different conditions against frequency. The thick solid line histogram depicts the original distribution as presented in Plaschke et al. (2009b). The hatched area shows the same distribution reduced by those events for which no SW data from the OMNI dataset were available. Weighting of the events to approximate SW parameter distributions shown in Fig. 2 yields the distribution depicted by the yellow bars. For this case the probability density function (normalized for integration in the interval between 0 and $6 \mathrm{mHz}$ ) is indicated by the right axis.

as introduced by Samson et al. as well as to the bin corresponding to the low frequency continuation of the regularly spaced frequency series $(0.7 \mathrm{mHz})$. Each of these numbers was divided by the total number of events in the whole frequency interval $(0$ to $6 \mathrm{mHz})$. This quantity $\left(P_{\mathrm{MF}}\right)$ is the probability for the MP to oscillate with the fundamental (or so-called "magic") frequencies mentioned above under the given SW conditions at the specified longitude sector. The procedure is applied to unweighted and weighted events to allow for a comparison of the results.

These are depicted in Fig. 4. The solid line in both top (weighted events) and bottom panels (unweighted events) shows the probability of occurrence for MP oscillations at the fundamental frequencies $\left(P_{\mathrm{MF}}\right)$. The combinations of applied event selection criteria have been sorted by this quantity, which increases monotonically towards the right side of the diagrams. The color schemes below them display these corresponding combinations of criteria. Their application as specified left of the schemes is indicated by a green box; the application of the opposite criterion is shown by a red box. Bars within the diagrams indicate the total number of events selected within the frequency range between 0 and $6 \mathrm{mHz}$. Combinations of criteria resulting in less than 60 events selected ( 2 per bin on average) were dismissed, because $P_{\mathrm{MF}}$ may not be computed accurately any more if too few events contribute to the underlying frequency distribution.

As can be clearly seen, the green boxes tend to be concentrated on the right side in both top and bottom panels. Consequently, the conditions $B_{z}>0 \mathrm{nT}, v_{\mathrm{sw}}<500 \mathrm{~km} / \mathrm{s},|\lambda|<40^{\circ}$
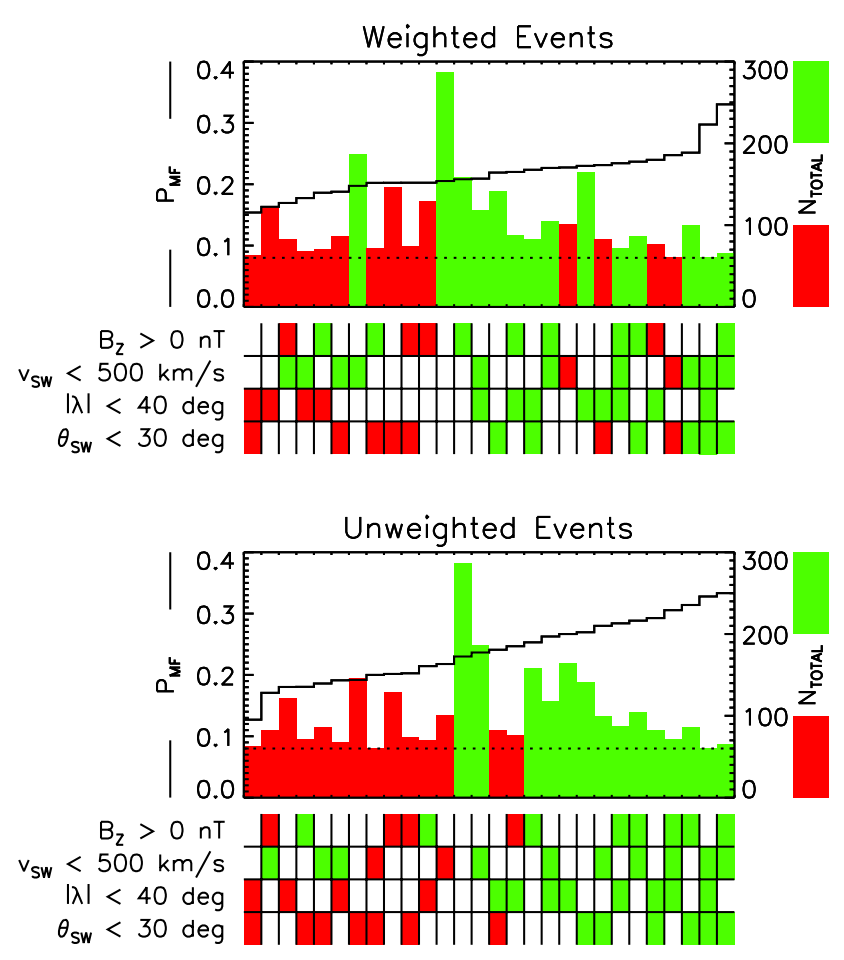

Fig. 4. Probability of occurrence of weighted (top panel) and unweighted (bottom panel) MP oscillation events with the frequencies $0.7,1.3,1.9,2.7$ and $3.3 \mathrm{mHz}$ normalized to all MP oscillation events within the frequency band 0 to $6 \mathrm{mHz}\left(P_{\mathrm{MF}}\right.$, solid line, left axis). Below each diagram the corresponding criteria applied for event selection are shown: green indicates its application, red the application of the opposite criterion. If unconsidered the corresponding block is left white. Bars in the diagrams show the number of events selected (right axis). 60 has been chosen as lower limit (dotted line). Red bars indicate that one or more criteria have been applied in the opposite sense to the one specified.

and $\vartheta_{\text {sw }}<30^{\circ}$ correspond to higher probabilities of occurrence $P_{\mathrm{MF}}$. Interestingly, there is a difference in slope of the $P_{\mathrm{MF}}$ graphs obtained from weighted and unweighted event frequency distributions: the former one is over large parts much shallower. Only the last two $P_{\mathrm{MF}}$ data points (on the right edge) appear significantly increased; the probability of occurrence of MP oscillation events at the fundamental frequencies selected with the corresponding criteria is highest in both panels. This indicates that only if several of the above stated conditions are fulfilled simultaneously, the excitation of the MP with the frequencies under consideration is significantly enhanced.

In row (group) 8 of Table 1 basic and sample population probabilities of occurrence for events observed under these favorable conditions can be found $\left(P_{\mathrm{b}}=0.031\right.$ and $\left.P_{\mathrm{S}}=0.144\right)$. It is noticeable that $P_{\mathrm{S}}$ of this group is by far the highest; the absolute difference between both probabilities is also the largest. Hence, the weighting factor for the events 


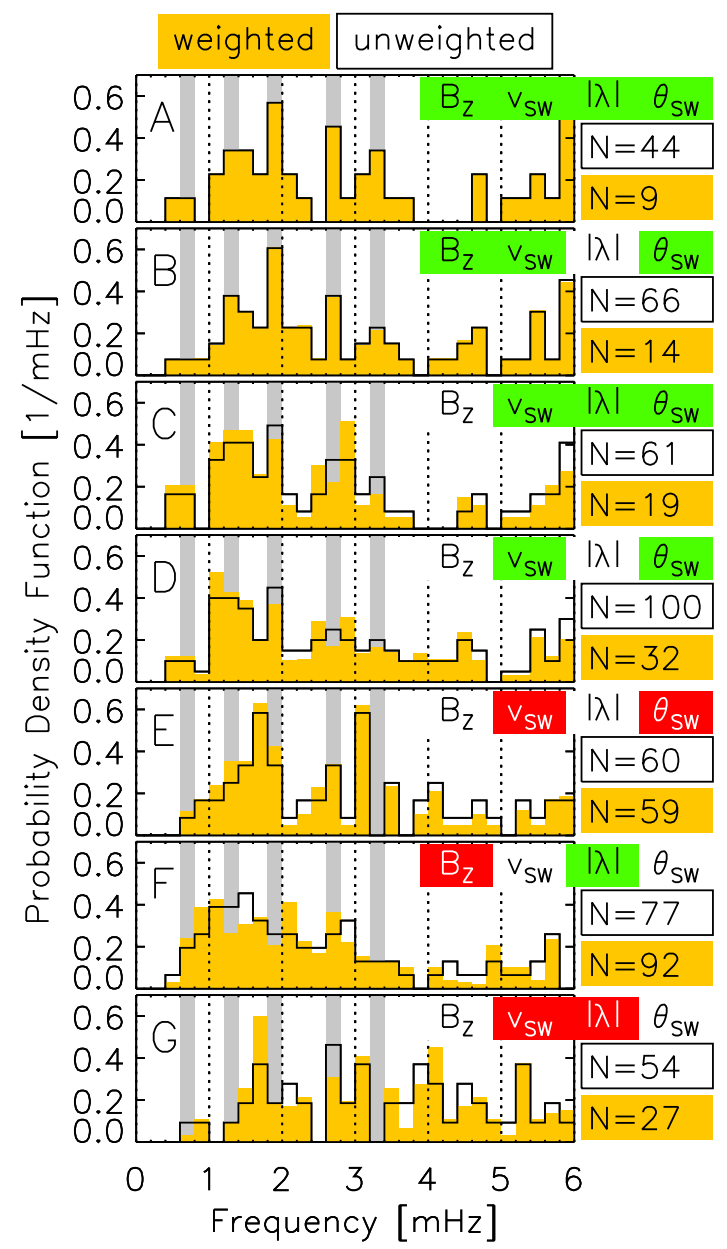

\begin{tabular}{ccc}
\hline $\mathrm{B}_{\mathrm{Z}}:$ & $>0 \mathrm{nT}$ & $<0 \mathrm{nT}$ \\
$\mathrm{v}_{\mathrm{sw}}:$ & $<500 \mathrm{~km} / \mathrm{s}$ & $>500 \mathrm{~km} / \mathrm{s}$ \\
$|\lambda|:$ & $<40 \mathrm{deg}$ & $>40 \mathrm{deg}$ \\
$\theta_{\mathrm{sw}}:$ & $<30 \mathrm{deg}$ & $>30 \mathrm{deg}$
\end{tabular}

Fig. 5. Probability density functions of MP oscillation frequencies in the interval between 0 and $6 \mathrm{mHz}$ derived from unweighted (solid lines) and weighted (yellow bars) events. The conditions applied are given in the upper right corners of each panel (A) to (G). The color code indicates the condition with regard to each parameter according to the legend given below all panels. No green or red background indicates, that no selection of events was performed with regard to the specific parameter. Numbers $(N)$ on the right side of each panel show the (real/fictitious) numbers of (unweighted/weighted) events contributing to the corresponding distribution. Grey bars display the positions of the fundamental frequency bins.

of this characteristic is among the smallest. This shows that events of this group are in particular heavily overrepresented in the sample population and in the original dataset of MP oscillations. It is, hence, not surprising that their frequency maxima stick out even in the general MP frequency distribution discussed in Plaschke et al. (2009b).
Subsequent application of the aforementioned criteria gradually increases the relative number of fundamental frequency events as can be seen in the graph of $P_{\mathrm{MF}}$ for unweighted event frequency distributions (bottom panel of Fig. 4). By this process a background of events in the frequency distribution, which lacks in characteristic frequency maxima, is progressively removed. Hence, the more conditions are fulfilled, the higher probability $P_{\mathrm{MF}}$ is obtained, particularly if three (or more) criteria are applied (see rightmost part of both panels of Fig. 4). Unfortunately, less than 60 events are within group 8 (between 0 and $6 \mathrm{mHz}$ ), and thus, its corresponding occurrence probability $P_{\mathrm{MF}}=0.364$ is not displayed in this figure, which is larger than any of the values of this quantity shown there.

As known from Fig. 2 sample population events are biased towards lower cone angles. Weighting of events is intended to counteract any bias in the sample population with regard to the basic parameter distributions. If the MP oscillation events at the fundamental frequencies are found to be clustered in one specific group, and if these events are not overrepresented in the sample population or its relative importance has been reduced by proper weighting, subsequent application of criteria towards the ones applying for the group of interest may lead to a less gradual increase in the probability of occurrence $P_{\mathrm{MF}}$, because other frequency enhancements (corresponding to other conditions) may be equally important and mask the fundamental frequency enhancements. As a result most combinations of criteria applied for the selection of weighted events will result in almost equal probabilities of occurrence for MP oscillations with these frequencies, even if adverse conditions are applied (see red bars in the upper panel diagram of Fig. 4 among the green ones on the right side). Only when most of the criteria are applied together, the probability increases noticeably. This explains the shallower increase in $P_{\mathrm{MF}}$ for weighted event frequency PDFs in comparison to the more gradual increase observed for unweighted events as described before.

Initially, the question was posed if a dominant source mechanism for the "magic" frequency maxima in the frequency PDFs could be identified. At this stage it can be stated that a strong dependence of the PDFs on the examined SW parameters $B_{z}, v_{\mathrm{sw}}$ and $\theta_{\mathrm{sw}}$ is apparent. If periodic pressure perturbations (of typical length scales) in the SW were primarily accountable for the MP oscillations observed, an enhanced appearance of these periodic perturbations during times of the above stated SW conditions would be expected. Such a dependence has not been reported.

Figure 5 displays the frequency PDFs for some selected combinations of applied criteria. These criteria are given in the upper right corner of each panel (a to g) indicated by the color coding (see legend at the bottom part of the figure), which also corresponds to the one used in Fig. 4. Solid line/yellow bar histograms show the frequency PDFs computed from unweighted/weighted events. Numbers $(N)$ at the right of each panel indicate the numbers of events 
contributing to each PDF in the range between 0 and $6 \mathrm{mHz}$. In the case of the weighted events (yellow background) this number corresponds to a fictitious number given by the sum of all weighting factors of the corresponding events. Grey bars show the positions of the fundamental frequency bins at $0.7,1.3,1.9,2.7$ and $3.3 \mathrm{mHz}$.

Panel (a) corresponds to the events from row (group) 8 in Table 1; as can be seen in the figure these frequencies stick out extraordinarily well. The number of contributing events in this group is relatively high in the shown frequency range $(N=44)$, however, not sufficient to be considered for Fig. 4. The next panels (b) to (f) display frequency PDFs related to condition combinations, under which weighted events showed highest occurrence probabilities $P_{\mathrm{MF}}$ (upper panel of Fig. 4). It is evident that panels (a) and (b) exhibit maxima at the specific frequencies most prominently. For the PDFs of panel (b) only the restriction with respect to the longitude $\lambda$ has not been applied. Numbers of events, however, have not increased dramatically (from 44 to 66) indicating that fewer events have been observed at high flank conditions, while other conditions still apply. These are spread across the frequencies and, hence, do not disrupt the overall picture. The good agreement between unweighted and weighted event distributions (solid line and yellow bar histograms) results from most events belonging to one group and, therefore, weighting not having a great effect. For panel (a) this is strictly true; both distribution must and do agree.

Panels (c) and (d) differ from (a) and (b) with respect to the non-application of the IMF $B_{z}$ criterion. As a consequence the matching of maxima at the fundamental frequency positions is notably worse. The distributions obtained from unweighted events display broader maxima at the correct positions. For weighted events the PDFs differ more: the maximum at $2.7 \mathrm{mHz}$ is absent. This shows the dependence of the PDF maxima with respect to the constraint on IMF $B_{z}$. A few events measured under southward IMF conditions (where all other conditions coincide) are able to disturb the distribution. Taking further into account that for both unweighted and weighted event distributions selection of only southward events (without any other condition applied) yields less probability of occurrence $P_{\mathrm{MF}}$ than if no selection takes place (see Fig. 4), we have to conclude that $B_{z}>0 \mathrm{nT}$ is a necessary ingredient for the appearance of prominent MP oscillations at the specified frequencies.

Thus, sources of MP oscillations dependent on southward IMF $B_{z}$ conditions for their appearance can be discarded as predominant sources. This applies, for instance, to periodic reconnection on the equatorial MP, which is known to be strongly dependent on the IMF $B_{z}$ direction. Flux transfer events (FTEs) are observed at the dayside noon sector almost always during southward IMF conditions (e.g., Russell and Elphic, 1978), when reconnection is enhanced. For flank sector FTEs the distribution with regard to IMF $B_{z}$ is somewhat more equilibrated; yet more FTE events are seen here at $B_{z}<0 \mathrm{nT}$ conditions as well (Kawano and Russell, 1997). FTE periodicities of a wider spectrum with an average of 8 min (Rijnbeek et al., 1984; Lockwood and Wild, 1993) have been reported, which caused them to be considered a quasiperiodic phenomenon: Reconnected flux tubes are dragged antisunward causing pulsations on the MP. However, our results showing that MP oscillations at the fundamental frequencies are primarily seen during times of northward IMF effectively rule out FTEs as their dominant source.

For computing the PDFs of panels (e), (f) and (g) of Fig. 5 event selection criteria unfavorable to the prominent development of MP oscillations at the fundamental frequencies have also been applied (red bars). As can be clearly seen, the distributions differ very much from the one displayed in panel (a). No prevalence for these frequencies can be found in these PDFs, neither for the weighted nor for the unweighted event distributions.

Events observed under conditions particularly favorable to the development of Kelvin-Helmholtz instability (KHI) waves on the MP have been selected to compute the frequency PDF shown in panel (g); these are high solar wind velocity and flank events, where the velocity shear driving possible KHI waves is larger. With the exception of the maximum at $2.7 \mathrm{mHz}$ other maxima of both distributions displayed do not agree with the fundamental frequency bins highlighted by the grey bars. Hence, this result may suggest that the KHI cannot be regarded as the major source for the appearance of the specific frequency maxima. It should be noted, however, that changing SW conditions after the initial growth phase of a KHI wave and later observation of this wave at a different location may lead to an erroneous interpretation of the wave source; the computed PDFs cannot contribute to ruling out this scenario.

Assuming the MP surface or Kruskal-Schwarzschild (KS) eigenmodes as described in Plaschke et al. (2009b) to be the primary origin of the observed peaks in the unconditioned PDF (see Fig. 3), enhancements at these frequencies should be visible in PDFs conditioned by northward IMF, low SW velocity and low longitude angle: The stability of the MP against reconnection during northward IMF should favour the unperturbed propagation of Alfvénic surface waves on it. This is also enhanced by low SW velocities, as known from KHI theory. Increased KS wave occurrence is expected around local noon due to bending of MP flux tubes at the flanks. Therefore, the conditions of northward $B_{z}$ and moderate or low SW velocity $v_{\text {sw }}$ seem to be specially favorable for the propagation of Alfvénic KS surface waves particularly on the dayside noon MP.

As can be seen in both Figs. 4 and 5, a low cone angle is among the favorable conditions for the development of MP oscillations at the fundamental frequencies. In contrast to the other three conditions this one may be related to the excitation of MP Alfvénic surface or Kruskal-Schwarzschild mode (KS) oscillations. A low IMF cone angle shifts the foreshock to the region upstream of the dayside noon sector 
bow shock, which then acts as an additional source of compressional disturbances for the MP in the same sector (see Fairfield et al., 1990). Normal IMF magnetic field conditions (low cone angle) could also be responsible for higher normal speed streams within the magnetosheath leading to local indentations of the MP, which would also have to be regarded as localized compressional disturbances. Since MP oscillations were predominantly observed during low cone angle conditions, although the basic population suggests that times of higher SW cone angle are much more probable (see bottom panel of Fig. 2), the question may be posed if these external localized compressional disturbances constitute a stronger source of MP oscillations than boundary instabilities of the MP itself (Shue et al., 2009; Hietala et al., 2009). As described in Glassmeier and Heppner (1992) these compressional disturbances disrupt the Chapman-Ferraro current system locally and may drive an Alfvénic surface wave via the closing field-aligned currents on the MP. The tension of the MP field lines would then act as restoring force transporting the indentations away in field-aligned direction.

The mechanism of resonant mode coupling of magnetosheath compressional and MP Alfvén or slow mode boundary surface waves has been extensively discussed by Belmont et al. (1995) and De Keyser et al. (1999) in the MHD approximation. They found four possible classes of solutions for incoming compressional waves: transmission of the wave into the magnetosphere or total reflection (evanescent wave on the magnetospheric side) combined with resonant mode coupling or its absence. Resonant mode conversion occurs when at any point inside the MP transition layer the Alfvén or slow mode resonance conditions are met locally such that energy can be transferred to these surface waves. The magnetic field configurations on both sides of the boundary (and other changing quantities across the boundary) determine the range of possible tangential wave vectors of incoming waves for which mode coupling should occur. An increasing shear angle between the magnetic fields (rotation across the MP) also extends this range for initial compressional waves; the likelihood of resonant mode coupling is increased. From this point of view a strictly northward pointing magnetosheath field may not be as favorable for the development of KS waves than a sheared magnetic field with a northward component on the magnetosheath side.

Finally, we would like to point out that all the above stated interpretations do not exclude the possibility of MP surface waves to be present also during conditions other than those mentioned to be favorable for their development. In these cases a potentially higher level of non KS wave MP fluctuations may be responsible for hiding them in the frequency spectra.

\section{Conclusions}

Comparing the SW parameter and longitude of observation distributions from the sample population with the basic popu- lation directly obtained from the OMNI SW dataset we found that except for the IMF cone angle the distributions generally agree. The MP oscillation half-period events observed took place mainly during times of low SW cone angle whereas the opposite (higher solar wind cone angle) is usually the case. This can be explained in terms of additional stability of the dayside MP when the magnetic field of the incident SW is already tangential to the boundaries upstream of the bow shock (dayside perpendicular shock).

The inequalities in the parameter distributions of sample and basic populations have to be taken into account in order to draw correct conclusions from the appearance of the MP oscillation frequency PDFs under different SW conditions. Otherwise overrepresented groups corresponding to certain parameter ranges tend to impose their distributions. This is done by weighting of events.

Computing frequency distributions from events selected by applying different criteria we found that the particular parameter combination of northward $B_{z}$, low or moderate SW speed $v_{\mathrm{sw}}$, low longitude angle $\lambda$ (dayside noon events) and low IMF cone angle $\vartheta_{\text {sw }}$ favors the development of MP oscillations or waves with the fundamental (or so-called "magic") frequencies suggested by Samson et al. (1992) among others. The probability of occurrence of MP oscillations with these frequencies increases significantly if several of the mentioned conditions are fulfilled. This is particularly visible from the weighted event distributions (see Fig. 4 top panel). Since the group of events observed under these conditions is overrepresented, the unconstrained frequency distribution also displays maxima at the corresponding positions.

It is, hence, reasonable to conclude that sources which are known to be predominantly active during SW conditions other than the above stated, cannot be the main source for the specific frequency enhancements observed, although it should be clear that a lot of different sources of MP motion act simultaneously on the MP and will contribute to the overall shape of the full and unconstrained MP oscillation frequency PDF.

SW periodic density or pressure variations directly driving the MP oscillations (Kepko et al., 2002) were not reported to occur more often during some specific SW conditions. From this perspective they may also be disregarded as the primary source of the frequency enhancements observed due to the found SW dependence. However, their exclusion may be premature, since it is not clear if and how their appearance (with certain frequencies) may be dependent on the prevalent SW conditions. Further investigation on possible source mechanisms of these perturbations is needed to determine a possible dependence.

The KHI, which is known to be most significantly generated during periods of high solar wind velocity at the flanks of the MP (opposed conditions to the ones found to be favorable for fundamental frequency MP oscillations) do not seem to be the major source; the fact, that the frequency PDFs of KHI favorable conditions do not present any 
particular enhancements at the fundamental frequencies, also contributes to this conclusion. With higher certainty periodic reconnection or FTEs may be ruled out as main source for MP oscillations observed at these frequencies, because these are almost always observed during southward IMF.

Nevertheless, there are other sources acting on the MP not further considered here, for instance changes in the inner magnetospheric ring current, which change the position of the MP, or also cavity or waveguide modes, which might have a repercussion on the MP position. However, it is difficult to assign these internal magnetospheric sources of MP motion specific SW parameter conditions most favorable for their development. Yet they may not be excluded as possible sources for oscillations at the fundamental frequencies. In particular, cavity modes have been reported to be responsible for the development of standing inner magnetospheric Alfvén waves at the fundamental or "magic" frequencies. As a volume oscillator they would be capable of providing a frequency selection. However, in our case it might be difficult to explain, for instance, why the generation of these cavity modes should be dependent on IMF $B_{z}$.

Instead the combination of parameters for which maxima at the fundamental frequencies appear most enhanced is also favorable for the development of Kruskal-Schwarzschild eigenmodes of the MP. From this point of view they might be regarded as the most important source of these enhancements. Our results constitute, hence, indirect evidence for the existence of the Kruskal-Schwarzschild (KS) eigenmodes excited via localized compressional disturbances on the MP, although other sources occurring at similar conditions may also be responsible. For a more definitive answer on the question of their existence a more detailed analysis on a case study basis in needed, which shall include a careful wave vector determination of observed surface waves and a comparison of spacecraft measurements at the MP and ground based observations with theoretical predictions.

Acknowledgements. The IGEP team was financially supported by the German Zentrum für Luft- und Raumfahrt under grants 50QP0402 and 50OC0901. THEMIS was made possible by NASA, under contract NAS5-02099. The OMNI data were obtained from the GSFC/SPDF OMNIWeb interface at http://omniweb.gsfc.nasa. gov. We acknowledge J. P. McFadden for the use of THEMIS Electrostatic Analyzer (ESA) particle data.

Topical Editor I. A. Daglis thanks J. De Keyser and another anonymous referee for their help in evaluating this paper.

\section{References}

Angelopoulos, V.: The THEMIS Mission, Space Sci. Rev., 141, 534, doi:10.1007/s11214-008-9336-1, 2008.

Aubry, M. P., Russell, C. T., and Kivelson, M. G.: Inward motion of the magnetopause before a substorm., J. Geophys. Res., 75, 7018-7031, doi:10.1029/JA075i034p07018, 1970.
Aubry, M. P., Kivelson, M. G., and Russell, C. T.: Motion and structure of the magnetopause., J. Geophys. Res, 76, 1673-1696, doi:10.1029/JA076i007p01673, 1971.

Auster, H. U., Glassmeier, K. H., Magnes, W., Aydogar, O., Baumjohann, W., Constantinescu, D., Fischer, D., Fornacon, K. H., Georgescu, E., Harvey, P., Hillenmaier, O., Kroth, R., Ludlam, M., Narita, Y., Nakamura, R., Okrafka, K., Plaschke, F., Richter, I., Schwarzl, H., Stoll, B., Valavanoglou, A., and Wiedemann, M.: The THEMIS Fluxgate Magnetometer, Space Sci. Rev., 141, 235-264, doi:10.1007/s11214-008-9365-9, 2008.

Belmont, G., Reberac, F., and Rezeau, L.: Resonant amplification of magnetosheath MHD fluctuations at the magnetopause, Geophys. Res. Lett., 22, 295-298, doi:10.1029/94GL03078, 1995.

Berchem, J. and Russell, C. T.: The thickness of the magnetopause current layer - ISEE 1 and 2 observations, J. Geophys. Res., 87, 2108-2114, doi:10.1029/JA087iA04p02108, 1982.

Cline, A. K.: Six Subprograms for Curve Fitting Using Splines Under Tension, Commun ACM, 17, 220-223, 1974.

De Keyser, J.: The Earth's Magnetopause: Reconstruction of Motion and Structure, Space Sci. Rev., 121, 225-235, doi:10.1007/ s11214-006-6731-3, 2005.

De Keyser, J. and Čadež, V.: Excitation of low-frequency fluctuations at the magnetopause by intermittent broadband magnetosheath waves, J. Geophys. Res., 106, 29467-29477, doi: 10.1029/2001JA900078, 2001.

De Keyser, J., Roth, M., Reberac, F., Rezeau, L., and Belmont, G.: Resonant amplification of MHD waves in realistic subsolar magnetopause configurations, J. Geophys. Res., 104, 23992409, doi:10.1029/1998JA900060, 1999.

Elphic, R. C. and Russell, C. T.: ISEE-1 and 2 magnetometer observations of the magnetopause, in: Magnetospheric Boundary Layers, edited by Lemaire, J., vol. 148 of ESA Special Publication, pp. 43-50, 1979.

Fairfield, D. H.: Average and unusual locations for the earth's magnetopause and bow shock., J. Geophys. Res., 76, 6700-6716, doi: 10.1029/JA076i028p06700, 1971.

Fairfield, D. H., Baumjohann, W., Paschmann, G., Luehr, H., and Sibeck, D. G.: Upstream pressure variations associated with the bow shock and their effects on the magnetosphere, J. Geophys. Res., 95, 3773-3786, doi:10.1029/JA095iA04p03773, 1990.

Francia, P. and Villante, U.: Some evidence of ground power enhancements at frequencies of global magnetospheric modes at low latitude, Ann. Geophys., 15, 17-23, 1997, http://www.ann-geophys.net/15/17/1997/.

Fujita, S., Glassmeier, K. H., and Kamide, K.: MHD waves generated by the Kelvin-Helmholtz instability in a nonuniform magnetosphere, J. Geophys. Res., 101, 27317-27326, doi:10.1029/ 96JA02676, 1996.

Glassmeier, K.-H. and Heppner, C.: Traveling magnetospheric convection twin vortices - Another case study, global characteristics, and a model, J. Geophys. Res., 97, 3977-3992, doi: 10.1029/91JA02464, 1992.

Glassmeier, K.-H., Auster, H.-U., Constantinescu, D., Fornaçon, K.-H., Narita, Y., Plaschke, F., Angelopoulos, V., Georgescu, E., Baumjohann, W., Magnes, W., Nakamura, R., Carlson, C. W., Frey, S., McFadden, J. P., Phan, T., Mann, I., Rae, I. J., and Vogt, J.: Magnetospheric quasi-static response to the dynamic magnetosheath: A THEMIS case study, Geophys. Res. Lett., 35, L17S01, doi:10.1029/2008GL033469, 2008. 
Haaland, S. E., Sonnerup, B. U. Ö., Dunlop, M. W., Balogh, A., Georgescu, E., Hasegawa, H., Klecker, B., Paschmann, G., PuhlQuinn, P., Rème, H., Vaith, H., and Vaivads, A.: Four-spacecraft determination of magnetopause orientation, motion and thickness: comparison with results from single-spacecraft methods, Ann. Geophys., 22, 1347-1365, 2004,

http://www.ann-geophys.net/22/1347/2004/.

Harrold, B. G. and Samson, J. C.: Standing ULF modes of the magnetosphere - A theory, Geophys. Res. Lett., 19, 1811-1814, doi: 10.1029/92GL01802, 1992.

Hasegawa, A. and Chen, L.: Theory of Magnetic Pulsations, Space Sci. Rev., 16, 347-359, doi:10.1007/BF00171563, 1974.

Hietala, H., Laitinen, T. V., Andreeova, K., Vainio, R., Vaivads, A., Palmroth, M., Pulkkinen, T. I., Koskinen, H. E. J., Lucek, E. A., and Rème, H.: Supermagnetosonic Jets behind a Collisionless Quasiparallel Shock, Phys. Rev. Lett., 103, 245001, doi:10.1103/PhysRevLett.103.245001, 2009.

Kawano, H. and Russell, C. T.: Survey of flux transfer events observed with the ISEE 1 spacecraft: Dependence on the interplanetary magnetic field, J. Geophys. Res., 102, 11307-11314, doi: 10.1029/97JA00481, 1997.

Kepko, L. and Spence, H. E.: Observations of discrete, global magnetospheric oscillations directly driven by solar wind density variations, J. Geophys. Res., 108, 1257-1269, doi:10.1029/ 2002JA009676, 2003.

Kepko, L., Spence, H. E., and Singer, H. J.: ULF waves in the solar wind as direct drivers of magnetospheric pulsations, Geophys. Res. Lett., 29, 1197-1200, doi:10.1029/2001GL014405, 2002.

Khrabrov, A. V. and Sonnerup, B. U. Ö.: Orientation and motion of current layers: Minimization of the Faraday residue, Geophys. Res. Lett., 25, 2373-2376, doi:10.1029/98GL51784, 1998.

Kruskal, M. and Schwarzschild, M.: Some instabilities of a completely ionized plasma, Proc. R. Soc. Lon., A223, 348-360, 1954.

Lockwood, M. and Wild, M. N.: On the quasi-periodic nature of magnetopause flux transfer events, J. Geophys. Res., 98, 59355940, doi:10.1029/92JA02375, 1993.

McFadden, J. P., Carlson, C. W., Larson, D., Ludlam, M., Abiad, R., Elliott, B., Turin, P., Marckwordt, M., and Angelopoulos, V.: The THEMIS ESA Plasma Instrument and Inflight Calibration, Space Sci. Rev., 141, 277-302, doi:10.1007/ s11214-008-9440-2, 2008.

Paschmann, G., Haaland, S., Sonnerup, B. U. Ö., Hasegawa, H., Georgescu, E., Klecker, B., Phan, T. D., Rme, H., and Vaivads, A.: Characteristics of the near-tail dawn magnetopause and boundary layer, Ann. Geophys., 23, 1481-1497, 2005, http://www.ann-geophys.net/23/1481/2005/.

Phan, T. D. and Paschmann, G.: Low-latitude dayside magnetopause and boundary layer for high magnetic shear 1 . Structure and motion, J. Geophys. Res., 101, 7801-7816, doi:10.1029/ 95JA03752, 1996.

Plaschke, F., Glassmeier, K.-H., Auster, H. U., Angelopoulos, V., Constantinescu, O. D., Fornaçon, K.-H., Georgescu, E., Magnes, W., McFadden, J. P., and Nakamura, R.: Statistical study of the magnetopause motion: First results from THEMIS, J. Geophys. Res., 114, A00C10, doi:10.1029/2008JA013423, 2009a.

Plaschke, F., Glassmeier, K.-H., Auster, H. U., Constantinescu, O. D., Magnes, W., Angelopoulos, V., Sibeck, D. G., and McFadden, J. P.: Standing Alfvén waves at the magnetopause, Geophys.
Res. Lett., 36, L02104, doi:10.1029/2008GL036411, 2009 b.

Rezeau, L., Morane, A., Perraut, S., Roux, A., and Schmidt, R.: Characterization of Alfvenic fluctuations in the magnetopause boundary layer, J. Geophys. Res., 94, 101-110, doi:10.1029/ JA094iA01p00101, 1989.

Rijnbeek, R. P., Cowley, S. W. H., Southwood, D. J., and Russell, C. T.: A survey of dayside flux transfer events observed by ISEE 1 and 2 magnetometers, J. Geophys. Res., 89, 786-800, doi:10. 1029/JA089iA02p00786, 1984.

Russell, C. T. and Elphic, R. C.: Initial ISEE magnetometer results - Magnetopause observations, Space Sci. Rev., 22, 681-715, doi: 10.1007/BF00212619, 1978.

Samson, J. C., Harrold, B. G., Ruohoniemi, J. M., Greenwald, R. A., and Walker, A. D. M.: Field line resonances associated with MHD waveguides in the magnetosphere, Geophys. Res. Lett., 19, 441-444, doi:10.1029/92GL00116, 1992.

Sarafopoulos, D. V.: A case study testing the cavity mode model of the magnetosphere, Ann. Geophys., 23, 1867-1880, 2005, http://www.ann-geophys.net/23/1867/2005/.

Shue, J., Chao, J., Song, P., McFadden, J. P., Suvorova, A., Angelopoulos, V., Glassmeier, K. H., and Plaschke, F.: Anomalous magnetosheath flows and distorted subsolar magnetopause for radial interplanetary magnetic fields, Geophys. Res. Lett., 36, L18112, doi:10.1029/2009GL039842, 2009.

Shue, J.-H., Chao, J. K., Fu, H. C., Russell, C. T., Song, P., Khurana, K. K., and Singer, H. J.: A new functional form to study the solar wind control of the magnetopause size and shape, J. Geophys. Res., 102, 9497-9512, doi:10.1029/97JA00196, 1997.

Sibeck, D. G., Baumjohann, W., Elphic, R. C., Fairfield, D. H., and Fennell, J. F.: The magnetospheric response to 8-minute period strong-amplitude upstream pressure variations, J. Geophys. Res., 94, 2505-2519, doi:10.1029/JA094iA03p02505, 1989.

Sibeck, D. G., Lopez, R. E., and Roelof, E. C.: Solar wind control of the magnetopause shape, location, and motion, J. Geophys. Res., 96, 5489-5495, doi:10.1029/90JA02464, 1991.

Sibeck, D. G., Prech, L., Safrankova, J., and Nemecek, Z.: Twopoint measurements of the magnetopause: Interball observations, J. Geophys. Res., 105, 237-244, doi:10.1029/1999JA900390, 2000.

Song, P., Elphic, R. C., and Russell, C. T.: ISEE 1 and 2 observation of the oscillating magnetopause, Geophys. Res. Lett., 15, 744747, doi:10.1029/GL015i008p00744, 1988.

Sonnerup, B. U. O. and Cahill Jr., L. J.: Magnetopause Structure and Attitude from Explorer 12 Observations, J. Geophys. Res., 72, 171-183, doi:10.1029/JZ072i001p00171, 1967.

Southwood, D. J.: The hydromagnetic stability of the magnetospheric boundary, Planet. Space Sci., 16, 587-605, doi:10.1016/ 0032-0633(68)90100-1, 1968.

Southwood, D. J.: Some features of field line resonances in the magnetosphere, Planet. Space Sci., 22, 483-491, doi:10.1016/ 0032-0633(74)90078-6, 1974.

Spreiter, J. R., Summers, A. L., and Alksne, A. Y.: Hydromagnetic flow around the magnetosphere, Planet. Space Sci., 14, 223-253, doi:10.1016/0032-0633(66)90124-3, 1966.

Viall, N. M., Kepko, L., and Spence, H. E.: Inherent length-scales of periodic solar wind number density structures, J. Geophys. Res., 113, A07101, doi:10.1029/2007JA012881, 2008. 